\title{
Neues Schablonensystem für die Implantologie präsentiert
}

Beim Bremer Implantologietag am 14.November 2015 stellte die BEGO Implant Systems dem Fachpublikum ihre Eigenfertigung der Bohrschablonen für die navigierte Chirurgie vor. Die Bohrschablonen werden am Standort Bremen mit modernster 3DDruck-Technologie hergestellt. Gleichzeitig steht den Anwendern für die Planung der Implantatpositionen das BEGO Guide Planungszentrum zur Verfügung. Eine eigene Planungssoftware wird nicht benötigt. Für den Datenaustausch steht das neue „BEGO Guide Orderportal“ unter guide.bego.com zur Verfügung. Interessierte Anwender können sich online registrieren. Zur Bestellung einer Schablone werden ein DVT und ein Gipsmodell benötigt. Beides wird zur BEGO nach Bremen geschickt. Die Daten werden aufbereitet und ein Pla-

nungsvorschlag erstellt.

Nach einer Pressemitteilung der

BEGO Implant Systems GmbH \& Co. KG,

Bremen

www.bego-implantology.com 